\title{
Generalization of the RIN Result to Heterogeneous Networks of Aggregate Schedulers and Leaky Bucket Constrained Flows
}

\author{
Gianluca Rizzo \\ EPFL, CH-1015 Lausanne, Switzerland \\ Email: gianluca.rizzo@epfl.ch
}

\author{
Jean-Yves Le Boudec \\ EPFL, CH-1015 Lausanne, Switzerland \\ Email: jean-yves.leboudec@epfl.ch
}

\begin{abstract}
We consider networks of FIFO aggregate schedulers. Quite surprisingly, the natural condition (node utilization inferior to one) in general is not sufficient in these networks to ensure stability (boundedness of delay and backlog at each node). Deriving good sufficient conditions for stability and delay bounds for these networks is of fundamental importance if we want to offer quality of service guarantees in such networks as DiffServ networks, high speed switches and network-on-chips. The main existing sufficient conditions for stability in these networks are the "DiffServ bound" [1] and the Route Interference Number (RIN) result [2]. We use an algebraic approach. First, we develop a model of the network as a dynamical system, and we show how the problem can be reduced to properties of the state transition function. Second, we obtain new sufficient conditions for stability valid without any of the restrictions of the "RIN result". We show that in practical cases, when flows are leaky bucket constrained, the new sufficient conditions perform better than existing results. We also prove that the "RIN result" can be derived as a special case from our approach. We finally derive an expression for a bound to delay at all nodes.
\end{abstract}

Keywords: Differentiated Services; QoS; Stability; Network Calculus

\section{INTRODUCTION}

One of the most widely deployed solutions to the problem of the scalability of scheduling policies is aggregate scheduling: that is, the scheduling decision at a node does not take into account which flow packets belong to, so that all packets are served as if they belonged to the same input flow. Scalability comes from the fact that no per-flow information needs to be stored. This scheduling policy is deployed in DiffServ networks and finds application also in high speed switches and network-on-chip systems.

We focus here on one of the most widely used scheduling algorithms, First-In-First-Out (FIFO), and we consider networks in which flows are constrained by arrival curves. One of the problems in this network model is stability, defined as boundedness over time of maximum packet delay and to queue size at each node [3]. Some examples of instability have been derived by Andrews in [4] and [5]. In particular, for leaky bucket constrained flows, ref. [5] brings examples of networks that are unstable at arbitrarily small network loads.

Some positive results exist on stability for these networks: Bramson [6], [7] [8] and Gamarnik [9] prove that all FIFO networks are stable if fresh input flows are constrained between $\sigma+\rho t$ and $-\sigma+\rho t$. Therefore, it seems that instability can arise when temporarily decreasing arrival rates.

Another positive result is in [10], where it is proved the stability of ring networks. Like feed forward networks, ring networks are always stable, provided that node stability is guaranteed. One of the main existing results is in [2], [11]: It assumes that flows are constrained by staircase arrival curves, and it is based on the homogeneous network model: packets all of the same size, nodes all with the same service rate, all packet arrival and departure times synchronous at all nodes. The derivation of the result is based on the concept of super chain, which models how packet interactions affect end-to-end packet delay. It also makes use of the concept of route interference number (RIN) of a flow, defined as the total number of flows that the considered flow meets along its path, counted with multiplicity if a flow interferes more then once. It introduces a sufficient condition for stability, in terms of lower bounds to the period of staircase arrival curves: For each flow, this period must be not larger than $(R I N+1)^{-1}$, where $R I N$ is the route interference number for that flow. It also derives simple formulas for backlog and delay bounds at all nodes. The main positive aspect of this result is that it does not depend on any topology; but it relies 
on assumptions that make it not useful in many practical cases. In what follows, we will call this result the "RIN result”.

In [12] Otel derives a generalization of the "RIN result" to heterogeneous networks. This result is based on a sufficient condition on stability in form of a minimum packet inter-arrival time for each flow. This way of shaping input traffic in a network is not compatible with constraints given in the form of arrival curves, which are the most commonly used in the majority of network models. That is, this sufficient condition cannot be cast into an arrival curve constraint. This makes the result not very useful in practical cases. Moreover, in the sufficient condition in [12] the minimum packet inter-arrival time for each flow scales linearly with the maximum packet size (or burst size) among all flows in the network. As an example, in a network where all flows satisfy the sufficient condition in [12] with a maximum packet size of 60 bytes, increasing the maximum packet size to 1500 bytes would decrease the maximum network utilization by a factor of 25 .

For networks with leaky bucket constrained flows, the main existing result is the one derived in [1]. It establishes a sufficient condition for stability in terms of a bound to the maximum node utilization in a network, in function of the maximum flow hop count $h$ in that network: If the network utilization is inferior to $(h-1)^{-1}$, then delay bounds can be derived at all nodes. This is again a result which is not relative to any topology, but as in practical cases $h$ can take quite large values (more than 20 for IP networks [13]), this condition leads to very low values of node utilization in realistic scenarios, and is therefore of little practical utility. In the rest of the paper, we will refer to this result as the "DiffServ bound".

The main limits of many existing results stem from the fact that their derivation is based on strong simplifying assumptions on the network model. In this paper we avoid those simplifying assumptions: We consider networks of generic topology, with packets of different sizes and links of different rates, with flows constrained by generic arrival curves, and we model the network as a dynamical system, clocked by events (packet arrivals and departures). We use the concept of super chain, introduced in [2]: The state variables are the maximum number of bytes present in a super chain relative to a given flow. The first main contribution of this paper is the derivation of an iterative mapping, based on an operator $F$ (given in detail in Section III) that represents the state transition function of the network, modeled as a dynamical system; we also prove how the problem of stability can be reduced to properties of this operator. The second main contribution consists in the derivation of sufficient conditions for stability, in terms of upper bounds to flow rates. We show that in the case where flows are leaky bucket constrained, the new sufficient conditions perform radically better than the previously existing results. Our results improve over the "RIN result" by generalizing it to networks with leaky bucket constrained flows, with nodes of different service rates, and packets of different sizes. We show that in the homogeneous network setting, the new bounds are at least as good as those in the "RIN result". We finally derive an expression for a bound to delay at all nodes.

The paper is organized as follows: in Section II we introduce the network model and the main concepts used in the present paper, and we define the states' space. In Section III we present an operator that represents the state transition function of our network, and we characterize the evolution over time of the state variables, showing how stability properties of the network are associated to the properties of the operator $F$.

In Section IV we derive the new sufficient stability condition. Finally, in Section VI the new results are assessed on a set of networks, and their performance compared to existing results.

\section{MODEL AND ASSUmptions}

\section{A. Network Model}

We assume the traffic in the network is organized in flows: each flow $f$ is constrained by a leaky bucket arrival curve, of the form $\sigma_{f}+\rho_{f} t$, and in general it has packets of different size. We assume for each flow there exists a finite set of possible packet sizes. We consider a network whose nodes are store-and-forward, FIFO schedulers, that perform aggregate scheduling. Each node offers to the aggregate of flows a service curve of the rate-latency type $\beta_{r, T}(t)=r(t-T)^{+}$, with service rate $r$ and latency $T$, generally different for each node. We assume service curves are strict (i.e. during a busy period of duration $u$, the output of the system is at least $\beta_{r, T}(u)$ )[14]. This is a very general node model, encompassing many scheduling disciplines (e.g. priority schedulers, or FIFO constant rate schedulers).

With $\Delta_{n}$ we denote the propagation delay of the link at the output of node $n$. 
TABLE I

NOTATION USED IN THE PAPER.

\begin{tabular}{|c|l|}
\hline Symbol & Definition \\
\hline \hline $\mathcal{N}^{n}, N^{n}$ & Set of all flows passing through node $n$, of cardinality $N^{n}$ \\
\hline $\mathcal{N}$ & Set of all flows in the network \\
\hline path $(f)$ & Ordered sequence of nodes, constituted by all the nodes traversed by flow $f$ \\
\hline $\mathcal{I}(\underline{n})$ & Set of all the nodes in the ordered sequence of nodes $\underline{n}$; \\
\hline$\sigma_{f}+\rho_{f} t$ & $\begin{array}{l}\text { Arrival curve for flow } f \text { at the input to the network, with burstiness } \sigma_{f} \text { and sustainable } \\
\text { rate } \rho_{f}\end{array}$ \\
\hline $\mathcal{U}_{f}^{n}$ & Set of those nodes belonging to path $(f)$ that precede node $n$ on the path of flow $f$ \\
\hline $\mathcal{D}_{f}^{n}, D_{f}^{n}$ & $\begin{array}{l}\text { Set of all the flows that arrive at node } n \text { from the same node as flow } f \text {, of cardinality } \\
D_{f}^{n}\end{array}$ \\
\hline$a_{f}^{n}$ & Amount of flow $f$ 's bytes present in the queue at node $n$ at time 0. \\
\hline$r_{n}$ & Service rate of node $n$ \\
\hline$T_{n}$ & Latency of node $n$ \\
\hline$\Delta_{n}$ & Propagation delay \\
\hline$p r e c(n, f)$ & Node that precedes node $n$ on the path of flow $f$ \\
\hline$L_{f}$ & Maximum packet size for flow $f$ \\
\hline
\end{tabular}

We define a relevant network event as the dequeuing of a packet at a node. Starting from $t=0$, we consider the (ordered) succession of time instants $t_{p}, p \in \mathbb{N}$ associated to relevant network events in the considered network. $t_{p}$ denotes the time instant of the $p$-th network event. As we have a finite set of packet sizes for each flow, we have a finite number of network events in any finite time interval.

We assume that at time 0 at each node $n$ and for each flow $f$ passing from that node there are $a_{f}^{n} \geq 0$ bytes from flow $f$ in the queue.

\section{B. Definition of the State Variables}

In order to model the network as a dynamical system, a crucial aspect is the choice of the state variables of the analyzed system. Our choice is based on the concept of super chain, first defined in [2]. Before describing in detail our definition of state variables, we recall here some definitions from [2][14]:

Definition 2.1: Given two packets $c$ and $d$, and a node $n$, we say that $c \preccurlyeq_{n} d$ if $c$ and $d$ are in the same busy period at $n$, and $c$ leaves $n$ before $d$.

Definition 2.2: Consider a sequence of packets $\underline{c}=\left(c_{0}, \ldots, c_{K}\right)$ and a sequence of nodes $\underline{n}=\left(n_{1}, \ldots, n_{K}\right)$ (all different). We say that $(\underline{c}, \underline{n})$ is a super chain if

- nodes $n_{1}, \ldots, n_{K}$ are all on the path of packet $c_{0}$;

- $c_{j-1} \preccurlyeq n_{j} c_{j}, j=1, \ldots, K$;

- the path of packet $c_{j}$ from $n_{j}$ to $n_{j+1}$ is a subpath of the path of $c_{0}$.

We call the path of packet $c_{0}$ from $n_{1}$ to $n_{K}$ the path of the super chain.

We now introduce some more definitions: We define a super chain $(\underline{c}, \underline{n})$ in which the first packet $c_{0}$ belongs to flow $f$ as a super chain relative to flow $f$.

Definition 2.3: We say that a packet $c$ is inserted in a super chain $(\underline{c}, \underline{n})$ if either $c=c_{j}, j=0, \ldots, K$, or there exists an index $l=1, \ldots, K$, for which it holds $c_{l-1} \preccurlyeq n_{l} c \preccurlyeq n_{l} c_{l}$.

The choice of state variables made in this paper is the following: For any flow $f$ in the network, and for any time instant $t_{p}$, we consider the variable $m_{f}[p]$, that represents the maximum number of bytes $\in f$ that can be inserted in any super chain relative to flow $f$, up to time $t_{p}$.

We indicate with $\underline{m}[p]$ the array of all the variables $m_{f}[p]$ relative to all flows in the network, for the time instant $t_{p}$.

\section{Strongly Connected Components}

We consider the connectivity graph of the network: it constitutes a digraph, that can be partitioned in a set of strongly connected components. We have the following result [15], that allows to study the stability of each strongly connected subnet independently from all the others: 
Lemma 2.1: A network is stable if and only if all its strongly connected components are stable.

Proof:If a network is stable, then of course all its strongly connected components will be stable. The converse statement is derived from applying Propositions (1) of [16] in networks with leaky bucket constrained flows.

Therefore, in the remainder of the paper we consider networks composed of a single strongly connected subnet, as the extension to the general case follows immediately from Lemma 2.1.

\section{Stability is Related to the Properties of the Network's State Transition Function}

In this section we derive a model of the network as a dynamical system, and we derive the expression of the state transition function of the network, which describes the evolution over time of the state variables.

Theorem 3.1: For any integer $p>0$, we have:

$$
\left\{\begin{array}{l}
\underline{m}[p] \leq F(\underline{m}[p-1]) \\
\underline{m}[0] \leq \underline{L}
\end{array}\right.
$$

where $F$ is the operator defined by $F(\underline{x})=\underline{x}^{\prime}$, with

$x_{f}^{\prime} \leq \rho_{f}\left(\sum_{n \in \mathcal{I}(\operatorname{path}(f))}\left\{\frac{\max _{f^{\prime} \in \mathcal{D}_{f}^{n}} L_{f^{\prime}}+\sum_{f^{\prime} \in \mathcal{N}^{n}} a_{f^{\prime}}^{n}}{r_{n}}++T_{n}+\Delta_{n}\right\}+\sum_{f^{\prime}} x_{f^{\prime}} \sum_{\underline{n}^{\prime} \in \mathcal{G}\left(f, f^{\prime}\right)} S\left(\underline{n}^{\prime}\right)\right)+\sum_{n \in \mathcal{I}(\operatorname{path}(f))} a_{f}^{n}+\sigma_{f}$

where:

- $S(\underline{n})=\frac{1}{r_{n_{1}}}+\sum_{j=2}^{K}\left(\frac{1}{r_{n_{j}}}-\frac{1}{r_{n_{j-1}}}\right)^{+}$

- $\mathcal{G}\left(f, f^{\prime}\right)$ is the set of maximal common subpaths between flows $f$ and $f^{\prime}$.

- $\underline{L}$ is the array of the maximum packet sizes for each flow in the network.

The proof of this result is in Appendix A. Theorem 3.1 describes the evolution over time of $\underline{m}[p]$. Intuitively, if the value of all the state variables is bounded over time, then also for any flow $f$ in the network the maximum number of bytes that can be served at any node during a busy period (and therefore the duration of the busy period itself) will be bounded over time, and this implies the stability of the network.

The following result shows how we can derive from the properties of the state transition function in Theorem 3.1 the stability properties of the network:

Theorem 3.2: Let's consider leaky bucket constrained flows. Call $\underline{m}^{*}$ a solution of the fixed point problem $\underline{m}=$ $F(\underline{m})$, where $F$ is the operator defined in Theorem 3.1. Then if $\underline{m}^{*}$ is finite, the network is stable.

The proof of this result is in Appendix B. The derivation of delay bounds is based on the following result:

Theorem 3.3: If $\underline{m}^{*}$ is a finite solution of the fixed point problem in Theorem 3.2, then for each node $n$ in the network an upper bound to packet delay is given by

$$
\min _{f \in \mathcal{N}^{n}}\left\{\sum_{f^{\prime} \in \mathcal{N}^{n} \backslash \mathcal{D}_{f}^{n}} \frac{m_{f^{\prime}}^{*}}{r_{n}}+\sum_{f^{\prime} \in \mathcal{D}_{f}^{n}} m_{f^{\prime}}^{*}\left(\frac{1}{r_{n}}-\frac{1}{r_{\operatorname{prec}(n, f)}}\right)^{+}\right\}+\frac{\sum_{f^{\prime} \in \mathcal{N}^{n}} a_{f^{\prime}}^{n}+\max _{f^{\prime} \in \mathcal{N}^{n}} L_{f^{\prime}}}{r_{n}}+T_{n}+\Delta_{n}
$$

Proof:It derives immediately from Lemma A.1.

\section{Sufficient Stability Conditions Generalize the RiN Result to Real Network}

We introduce here one of the main results of the present paper, which consists in a sufficient condition for the stability of an aggregate scheduling network in which flows are leaky bucket constrained. This represents a case of particular interest in applications: as an example, the network model in the DiffServ framework is based on leaky bucket constrained flows.

Theorem 4.1 (Generalized Source Rate Condition): With the given assumptions on network, if flows are leaky bucket constrained, if for any flow $f$ (whose path we indicate with $\left(n_{1}, \ldots, n_{K}\right)$ ) its rate $\rho_{f}$ satisfies the condition: 


$$
\rho_{f}<\left[\frac{N^{n_{1}}}{r_{n_{1}}}+\sum_{j=2}^{K} \frac{N^{n_{j}}-D_{f}^{n_{j}}}{r_{n_{j}}}+D_{f}^{n_{j}}\left(\frac{1}{r_{n_{j}}}-\frac{1}{r_{n_{j-1}}}\right)^{+}\right]^{-1}
$$

then the network is stable.

Proof:When flows are leaky bucket constrained, the operator $F$ defined in Theorem 3.1 is linear. In this case the condition on the fixed point problem in Theorem 3.2 to have a finite solution is to have the spectral radius of the matrix of the system be inferior than one. A sufficient condition for this to be true is to have the matrix row sums smaller than one. By imposing this last condition we get (3).

One of the main features of Theorem 4.1 is that it generalizes the "RIN result", which can be entirely derived as a special case of Theorem 4.1:

Corollary 4.1: If we consider the homogeneous network model, assuming arrival curves for fresh flows are of the staircase type, and for any flow $f$ in the network the packet inter-arrival time $\tau_{f}$ satisfies the condition $\tau_{f} \geq R I N_{f}+1$, where $R I N_{f}$ is the route interference number of flow $f$ [2], then the network is stable.

Proof:We note that each flow constrained by a staircase arrival curve with period $\tau_{f}$ is also constrained by a leaky bucket arrival curve, with rate $\rho_{f}=\frac{1}{\tau_{f}}$. Then Corollary 4.1 descends from applying Theorem 4.1 with the homogeneous network settings.

We can therefore note that the sufficient stability conditions in Theorem 4.1 extend the "RIN result" to leaky bucket constrained flows and removes the constraints on network homogeneity. The quantity at the denominator of the bound in (3) can be interpreted as a generalization of the concept of route interference number (RIN) of a flow. The new sufficient conditions in (3) hold not for a whole network, but for each of its strongly connected components separately. When a flow $f$ traverses more then one strongly connected subnet, the condition on its rate $\rho_{f}$ is the tightest among all those derived from (3) for each strongly connected subnet that it traverses. Another difference with respect to the "RIN result" is that while that result is derived by implicitly assuming the network to be empty at $t=0$, the sufficient conditions derived here are valid independently of the buffer content at all nodes at $t=0$. Finally, we note that the new sufficient conditions in (3) hold also for staircase arrival curves, as each flow constrained by a staircase arrival curve with period $\tau$ is also leaky bucket constrained, with rate $\rho=\frac{1}{\tau}$.

\section{DisCUSSION OF TIGHTNESS}

For a generic network, determining the tightness of the sufficient conditions for stability in Theorem 4.1 is an open issue. However, we can get an idea of how those sufficient conditions perform by applying them to some network examples for which we know their stability behavior.

One example is given by feed-forward networks, known to be always stable, provided that node utilization is inferior to one. For these networks, each node represents a strongly connected component of the network. Therefore, sufficient conditions in Theorem 4.1, applied to each node in isolation, give a maximum node utilization of 1 .

Another example of a network that is known to be always stable is the ring [10], provided that node utilization is strictly smaller than one. In these networks, sufficient conditions in Theorem 4.1 perform differently according to the number of flows in the ring and to their path, and in general they impose tighter conditions on flow rates than those derived by the natural condition at each node. Another check of the tightness of the sufficient conditions in (3) can be done by applying them over the network described in [5] as an example of instability. We see that, with the assumptions in that example, the maximum node utilization is at least a factor of $K^{3}$ larger than the one we obtain when imposing on flow rates the sufficient conditions in (3), where $K \geq 2$ is a parameter relative to the size of the network and to the path length of a flow.

\section{Numerical Assessment of the Result}

An important feature of these results is their dependence on network topology. Therefore, in order to assess numerically the quality of the sufficient conditions in Theorem 4.1, we applied them over a set of $n$ networks. The physical topology of the networks was generated using the BRITE network generator [17], with a node degree not smaller than 2. Path flows were determined using the Dijkstra algorithm, and selected in order to have a final hop count distribution close to the experimental ones [13]. The distribution of link rates approximates the one from 


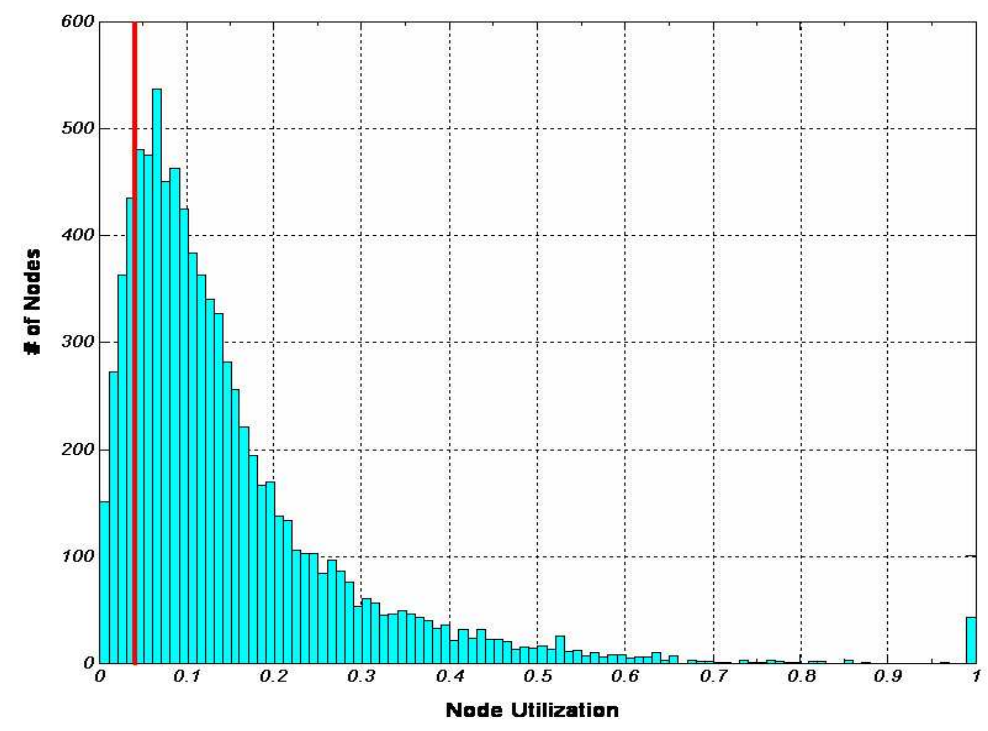

Fig. 1. Distribution of node utilization in 100 networks, with all flow rates equal to $99.5 \%$ of the bounds in Theorem 4.1 . The average node utilization is of 0.1460 , with a standard deviation of 0.1340 . The vertical line represents the maximum node utilization value that can be derived by applying the DiffServ bound [1], equal to 0.0455 for the considered networks. The new sufficient conditions for stability bring an average network utilization which is more than three times larger than the maximum node utilization that we can have by applying the DiffServ bound.

experimental data available in [18]. The resulting networks had a number of links between 80 and 100, a total number of flows between 180 and 200 an average hop count of 10.33, and a maximum hop count of 23. In Fig. 1 we have the empirical distribution of node utilization in the considered networks, where flow rates have been set to $99.5 \%$ of the value determined by bounds in (3).

We see that we have an average node utilization of 0.1460 , with a standard deviation of 0.1340 . The only other available result for this kind of network and flow arrival rates is the "DiffServ bound", that depends on the maximum hop count in the network (note that the "RIN result" does not apply in these network settings). Experimentally [13] it has been verified that the maximum hop count in a network can be larger than 23: so the "DiffServ bound" would bring to a maximum node utilization in the network of 0.0455 . Therefore, the new sufficient conditions for stability bring an average node utilization which is more than three times larger than the (maximum) node utilization that we can have by applying the "DiffServ bound".

\section{CONCLUSION}

In this paper we consider the problem of deriving good sufficient conditions for stability in networks of FIFO aggregate schedulers, when their connectivity graph has a generic topology. The main existing sufficient conditions for stability in these networks are either based on strong limiting assumptions on the network, and on ad-hoc proofs, difficult to generalize, or imply very low values of node utilization. In this paper we develop an algebraic approach to the problem, and we propose a model of the network as a dynamical system that does not rely on any limiting assumption on network and packet dynamics, and we show how the stability of the network is related to the properties of the state transition function. Hence we derive new sufficient conditions for stability, valid also for leaky bucket constrained flows and that hold without any of the restrictions on network and flows of the "RIN result". We show that in practical cases, in networks with a realistic hop count distribution the new sufficient conditions perform radically better than the main existing result that applies to these networks (the "DiffServ bound"), thus allowing for an average node utilization that is more than three times larger than the maximum value obtainable with the "DiffServ bound". We also prove that the "RIN result" can be derived as a special case from our approach. We finally derive an expression for delay bounds at all nodes. 


\section{REFERENCES}

[1] A. Charny and J.-Y. L. Boudec, "Delay bounds in a network with aggregate scheduling." in QofIS, 2000, pp. 1-13.

[2] I. Chlamtac, A. Faragó, H. Zhang, and A. Fumagalli, "A deterministic approach to the end-to-end analysis of packet flows in connection oriented networks," IEEE/ACM Transactions on Networking, vol. (6)4, pp. 422-431, 081998.

[3] T. Seidman, "First come, first served" can be unstable!" IEEE Transactions on Automatic Control, vol. 39, no. 10 , October 1994.

[4] M. Andrews, "Instability of fifo in session-oriented networks," in Eleventh Annual ACM-SIAM Symposium on Discrete Algorithms (SODA 2000), January 2000, pp. 440-447.

[5] — - "Instability of fifo in the permanent sessions model at arbitrarily small network loads," in ACM-SIAM Symposium on Discrete Algorithms (SODA 2007), New Orleans, USA, January 2007.

[6] M. Bramson, "Instability of fifo queueing networks," Annals of Applied Probability, vol. 4, no. 2, p. $414431,1994$.

[7] _ _ "Instability of fifo queueing networks with quick service times," Annals of Applied Probability, vol. 4, no. 3, p. 693 718, 1994.

[8] _ - "Stability of two families of queueing networks and a discussion of fluid limits," Queueing Syst., vol. 28, no. 1-3, pp. 7-31, 1998.

[9] D. Gamarnik, "Stability of adversarial queues via fluid models," Proc. of the 39th Annual Symposium on Foundations of Computer Science, p. 60 70, November 1998.

[10] L. Tassiulas and L. Georgiadis, "Any work-conserving policy stabilizes the ring with spatial re-use," IEEE/ACM Transactions on Networking, vol. 4, no. 2, pp. 205-208, Aug 1996.

[11] J.-Y. Le Boudec and G. Hebuterne, "Comment on a deterministic approach to the end-to-end analysis of packet flows in connection oriented network," IEEE/ACM Transactions on Networking, vol. 8, pp. 121-124, February 2000.

[12] F.-D. Otel and J.-Y. L. Boudec, "Deterministic end-to-end delay guarantees in a heterogeneous route interference environment." in QofIS, 2003, pp. 21-30.

[13] F. Begtasevi and C. V. Mieghem, "Measurements of the hopcount in internet," in Proceedings of Passive and Active Measurement (PAM2001), Amsterdam, April 2001.

[14] J.-Y. Le Boudec and P. Thiran, Network Calculus. A Theory of Deterministic Queueing Systems for the Internet. Springer Verlag LNCS, July 2001, vol. 2050.

[15] M.Blesa, "Deciding stability in packet-switched fifo networks under the adversarial queuing model in polynomial time," Proc. of the 19th International Symposium on Distributed Computing, LNCS Vol. 3724, pp. 429-441, 2005.

[16] B. Hajek, "Large bursts do not cause instability," IEEE Trans on Aut Control, vol. 45, pp. 116-118, Jan 2000.

[17] A. Medina, A. Lakhina, I. Matta, and J. Byers, "BRITE: Universal topology generation from a user's perspective, Tech. Rep. 2001-003, 12001.

[18] Swisscom, International Backbone Topology. (http://www.ip-plus.net/network/international.en.html), 2007.

\section{APPENDIX}

\section{A. Proof of Theorem 3.1}

Definition A.1: The super chain time is the time at which the last packet in the super chain is served at the last node of the super chain.

Also, with reference to Definition 2.2, at each node $n_{j}$ on the path of the super chain we call the packets $c_{j-1}$ and $c_{j}$ respectively open packet and close packet for that node.

Lemma A.1: The delay of a packet of flow $f$ that arrives at a node $n$ with service rate $r_{n}$ and latency $T_{n}$ on a link with rate $r^{\prime}$ is upper bounded by the quantity

$$
\frac{\Theta}{r_{n}}+\Gamma\left(\frac{1}{r_{n}}-\frac{1}{r^{\prime}}\right)^{+}+\frac{C+\max _{f^{\prime} \in \mathcal{D}_{f}^{n}} L_{f^{\prime}}}{r_{n}}+T_{n}+\Delta_{n}
$$

where $\Theta$ and $\Gamma$ and $C$ represent sum of packet sizes for packets served in the same busy period as the considered packet and before this packet. $\Theta$ and $\Gamma$ refer to packets arrived at the node respectively, on the same link as the considered packet and from other nodes or sources, while $C$ refers to packets present at the node at $t=0$.

Proof:The delay of the considered packet is the same it would experience in the case in which no packet arrives at the node before the beginning of the considered busy period and after the arrival of the considered packet: so we consider this case. Then the bound in (4) derives from computing the maximum horizontal distance between an aggregate arrival curve for all input flows of the form $\Theta+\max _{f^{\prime} \in \mathcal{D}_{f}^{n}} L_{f^{\prime}}+\min \left(\Gamma, r^{\prime} t\right)$, and the service curve $r_{n}\left(t-T_{n}-C / r_{n}\right)$, and including the constant delay $\Delta_{n}$.

Proof of Theorem 3.1: we proceed by induction on the index $p$ of relevant network events.

Base case: $\mathbf{p}=\mathbf{1}$. In order to upper bound the quantities $m_{f}[1]$, for any flow $f$, the worst case to consider is the one in which the first two relevant network events in the network are relative respectively to the first and the second packet served in the same busy period at a given node $n$.

Therefore we put in this case, and we consider the busy period that starts at node $n$ at $t=0$, in which the first 
and the second packets served belong respectively to flows $f$ and $f^{\prime}$. We have in this case a super chain relative to $f$ formed by two packets and a single node, $n . t_{1}$ is the time at which the second packet in the busy period is served. As it must be in the same busy period as the one served at $t_{0}$, then it must have arrived at the node by time $t_{0}\left(t_{0}\right.$ is the time after which the node starts serving the second packet, which should then be already at the node). As a consequence, taking into account those packets present at the node at $t=0$ too, we have for flow $f$ that $m_{f}[1] \leq \alpha_{f}\left(t_{0}\right)+a_{f}^{n}$. As $t_{0} \leq\left(m_{f}[0]+m_{f^{\prime}}[0]+L_{f}\right) / r_{n}+\Delta_{n}+T_{n}$, inequality in (2) holds.

Induction step. We try to find an upper bound to the variable $m_{f}[p]$. Let's consider a given super chain $(\underline{c}, \underline{n})$ relative to flow $f$ and with time $t_{q}, q \leq p$, and let's define the duration of this super chain the time interval since the emission time of $c_{0}$ (the first packet of this super chain) up to time $t_{q-1}$. The maximum number of flow $f$ 's bytes that can be inserted in this super chain is upper bounded by the maximum number of bytes from that flow that can be emitted during its duration (and not up to time $t_{q}$, as in order to be served by time $t_{q}$ or before at the last node of the super chain, a packet must arrive at that node by time $t_{q-1}$ ), plus all the flow $f$ 's packets present at $t=0$ in the network.

In what follows, we derive an upper bound to the duration of the considered super chain. For each node $n_{k}, k=$ $1, \ldots, K$ in the sequence of nodes path $(f)$, we indicate with $\Delta t_{i n t, k}$ the delay experienced at node $n_{k}$ by the open packet at that node (for nodes $\in \mathcal{I}(\operatorname{path}(f)) \backslash \mathcal{I}(\underline{n})$ we consider the open packet to coincide with the close packet). If $\Theta^{k}$ and $\Gamma^{k}$ represent sum of packet sizes for packets served in the same busy period as the open packet and before it, and coming from node $n_{k-1}$ (or from the same source as flow $f$ ), and from other nodes (or from fresh flows different than $f$ ), using Lemma A.1 we have for $k \geq 1$

$$
\Delta t_{i n t, k} \leq \frac{\Theta^{k}}{r_{n_{k}}}+\Gamma^{k}\left(\frac{1}{r_{n_{k}}}-\frac{1}{r_{p r e c}\left(n_{k}, f\right)}\right)^{+}+\frac{C_{k}^{\prime}+\max _{f^{\prime} \in \mathcal{D}_{f}^{n_{k}}} L_{f^{\prime}}}{r_{n_{k}}}+T_{n_{k}}+\Delta_{n_{k}}=B_{k}+T_{n_{k}}+\Delta_{n_{k}}
$$

where $C_{k}^{\prime}$ is relative to packets present at the node at $t=0$.

If $\Delta t_{k}$ is the time interval between the departure of the open packet and of the close packet, using the definition of strict service curve, we write

$$
\Delta t_{i n t, k}+\Delta t_{k} \leq B_{k}+T_{n_{k}}+\Delta_{n_{k}}+\frac{\gamma^{k}+\theta^{k}+C_{k}}{r_{n_{k}}}
$$

where $\gamma^{k}$ and $\theta^{k}$ are the sum of packet sizes for packets arrived at node $n_{k}$ respectively, from node $n_{k-1}$ (or from the same source as flow $f$ ), and from other nodes (or from fresh flows different than $f$ ), and inserted in the considered super chain, and $C_{k}$ is relative to packets present at the node at $t=0$. Therefore an upper bound to any super chain $(\underline{c}, \underline{n})$ relative to flow $f$ and with time time $t_{q}, q \leq p$ is obtained by summing up the bounds in (6) over all the nodes in the path of flow $f$. At each node $n_{k}$, each of the terms $\gamma^{k}, \theta^{k}, \Gamma^{k}, \Theta^{k}, C_{k}, C_{k}^{\prime}$ can be written as a sum of the contributions from all the input flows at the node. For instance, for $\Theta^{k}$ we can write $\Theta^{k}=\sum_{f^{\prime} \in \mathcal{N}^{n_{k}}} \Theta_{f^{\prime}}^{k}$. For any flow $f^{\prime}$ (not necessarily distinct from $f$ ), $\forall \underline{n}^{\prime}=\left(n_{l}, \ldots, n_{l+K^{\prime}}\right) \in \mathcal{G}\left(f, f^{\prime}\right)$, the contribution to the upper bound to the super chain duration is of the form

$$
\frac{\Theta_{f^{\prime}}^{l}+\theta_{f^{\prime}}^{l}}{r_{n_{l}^{\prime}}}+\sum_{k=l}^{l+K^{\prime}}\left[\Gamma_{f^{\prime}}^{k}\left(\frac{1}{r_{n_{k}^{\prime}}}-\frac{1}{r_{n_{k-1}^{\prime}}}\right)^{+}+\frac{\gamma_{f^{\prime}}^{k}+a_{f^{\prime}}^{n_{k}^{\prime}}}{r_{n_{k}^{\prime}}}\right]
$$

By definition of the variables $m_{f}[p]$, we have that $\left(\left(\ldots\left(\Theta_{f^{\prime}}^{l}+\theta_{f^{\prime}}^{l}+\Gamma_{f^{\prime}}^{l}+\gamma_{f^{\prime}}^{l}+\right) \vee \Gamma_{f^{\prime}}^{l+1}+\gamma_{f^{\prime}}^{l+1}\right) \vee \Gamma_{f^{\prime}}^{l+2}+\gamma_{f^{\prime}}^{l+2}\right) \vee \ldots \vee$ $\Gamma_{f^{\prime}}^{l+K^{\prime}}+\gamma_{f^{\prime}}^{l+K^{\prime}} \leq m_{f^{\prime}}[p]$. Therefore, the quantity in (7) is upper bounded by $m_{f^{\prime}}[p] S\left(\underline{n^{\prime}}\right)+\sum_{n^{\prime} \in \mathcal{I}\left(\underline{n}^{\prime}\right)} a_{f^{\prime}}^{n^{\prime}} / r_{n^{\prime}}$. Then for the considered super chain (as well as for any super chain relative to flow $f$ with time $\leq t_{p}$ ), putting together 
the contribution from all flows, we finally get the following upper bound to the super chain duration:

$$
\sum_{f^{\prime}} m_{f^{\prime}}[p] \sum_{\underline{n}^{\prime} \in \mathcal{G}\left(f, f^{\prime}\right)} S\left(\underline{n}^{\prime}\right)+\sum_{n \in \mathcal{I}(p a t h(f))}\left(\frac{\max _{f^{\prime} \in \mathcal{D}_{f}^{n}} L_{f^{\prime}}+\sum_{f^{\prime} \in \mathcal{N}^{n}} a_{f^{\prime}}^{n}}{r_{n}}+T_{n}+\Delta_{n}\right)
$$

Then using the expression of flow $f$ 's arrival curve, and taking into account those packets from flow $f$ present in the buffers in the network at $t=0$, we finally derive the upper bound in (2).

B. Proof of Theorem 3.2

Proof:As flows are leaky bucket constrained, the operator $F$ is linear, and takes the form $A \underline{x}+\underline{b}$ with $A$ a nonnegative matrix. For any flow $f$, the burstiness $\sigma_{f} \geq L_{f}$, therefore $\underline{b} \geq \underline{L}$. Therefore, any $\underline{m}^{*}$ that satisfies $\underline{m}^{*}=F\left(\underline{m}^{*}\right)$ is $\underline{m}^{*} \geq \underline{L}$. Now, as the mapping function $F$ is monotone increasing, we have for any integer $p>0, \underline{m}[p] \leq F(\underline{m}[p-1]) \leq F^{p}(\underline{m}[0]) \leq F^{p}(\underline{L}) \leq F^{p}\left(\underline{m}^{*}\right)=\underline{m}^{*}$. Then if $\underline{m}^{*}$ is finite, busy period duration at each node is bounded over time, and therefore also queue size and maximum packet delay. 